\title{
Survey on the Long Term Use of Antihypertensive Drug*
}

$\mathrm{M}$

ANY years have elapsed since various potent antihypertensive drugs were made available in medical practice and long term treatment of hypertensive patients were carried out in many clinics. The drug or drugs of choice in the hypertension treatment may alter from time to time according to the progress of pharmaceutics. However, it is worthwhile, at least as a milestone, to know the present status of hypertension treatment in Japan, e.g. what drug or drugs are commonly used, whether any beneficial effect on the objective or subjective findings are brought about or not. Thus the survey on the long term use of antihypertensive drug in Japan has been made in 1959 by the committee (chairman: S. Okinaka, M. D., Professor of Medicine, Faculty of Medicine, University of Tokyo) on the diagnosis, treatment and prevention of hypertension and cardiac diseases. This study was aided by a grant from the Welfare Ministry of Japan.

The members of the committee who participated in the study were:

Fuse, N., M.D., Vice President, Osaka National Hospital

Hirosawa, K., M.D., Assistant Professor, Tokyo Womens' Medical College

Ito, Y., M. D., Chief of Internal Medicine, Tokyo Second National Hospital

Kase, M., M.D., Ghief of Internal Medicine, Kanto Teishin Hospital Kato, T., M. D., President, Sendai National Hospital

Kobayashi, T., M.D., Professor of Medicine, Tokyo University Branch Hospital

Okinaka, S., M.D., Professor of Medicine, Tokyo University Hospital

Oshima, K., M.D., Professor of Medicine, Nihon University Hospital

Shigiya, R., M.D., Chief of Internal Medicine, Tokyo First National Hospital

Ueda, H., M.D., Professor of Medicine, Tokyo University Hospital

Wada, Y., M.D., Vice President, Nagoya National Hospital

The data were analyzed and described by M. Ikeda, M.D., Y. Ito, M.D. and T. Kobayashi, M.D. and assisted by H. Ohtsuka, B.S.N. and Y. Kojima, B. H. N., the University of Tokyo.

* Chairman: S. Okinaka, M.D., Professor of Medicine, Faculty of Medicine, University of Tokyo. 
The data were submitted by the following hospitals:

Dept. of Internal Medicine (Okinaka Clinic) Tokyo Univ. 32 cases

Dept. of Internal Medicine (Ueda Clinic) Tokyo Univ. 41 "

Dept. of Internal Medicine, Branch Hospital, Tokyo Univ. 34 ",

Dept. of Internal Medicine, Nihon University 43 "

Tokyo Womens' Medical College 79 "

Kanto Teishin Hospital $45 "$

Tokyo First National Hospital 13 ",

Tokyo Second National Hospital 20 "

Sendai National Hospital 44 "

Osaka National Hospital $\quad 46$ "

Nagoya National Hospital

Total $\frac{33 "}{430 \text { cases }}$

Among the data submitted on 430 cases, 379 cases were chosen as the group on the long term (over one year) use of antihypertensive drug.

The blood pressure value prior to the use of antihypertensive drug was classified according to the following criteria. Since the blood pressure value recorded prior to the use of drug cannot be ascertained as a sufficiently controlled one from the survey report alone, the values reported were used as such.

Classification according to systolic pressure

\begin{tabular}{lrllrl} 
Group I & systolic pressure & below & $179 \mathrm{~mm} . \mathrm{Hg}$ & 82 cases \\
Group II & systolic pressure & $180-219 \mathrm{~mm} . \mathrm{Hg}$ & 214 & $"$ \\
Group III & systolic pressure & above & $220 \mathrm{~mm} . \mathrm{Hg}$ & 83 & " \\
\cline { 3 - 5 } & & & Total & 379
\end{tabular}

Classification according to diastolic pressure

\begin{tabular}{|c|c|c|c|c|c|}
\hline Group & diastolic pressure & below & $99 \mathrm{~mm} . \mathrm{Hg}$ & 96 & cases \\
\hline Group II & diastolic pressure & $100-$ & $119 \mathrm{~mm} . \mathrm{Hg}$ & 183 & $"$ \\
\hline Group III & diastolic pressure & above & $120 \mathrm{~mm} . \mathrm{Hg}$ & 100 & $"$ \\
\hline & & & Total & 379 & \\
\hline
\end{tabular}

The group of hypertensive cases studied in this survey have all visited the out-patient department of a specific institution for over a period of one year at the minimum. Therefore, the cases which were unable to continue to visit the clinic because of the following reasons were not included. Case which developed serious complication at an early stage of the treatment; cases with various reason such as finance or family conditions; and cases which changed physicians because of lack of improvement of their condition in spite of the treatment. The fact that 
these cases were excluded from the study should be kept in mind in interpreting the results obtained through the survey.

1. The effect of antihypertensive drug in lowering the blood pressure.

In considering the effect of the antihypertensive drug, the blood pressure value recorded prior to and after the use of antihypertensive drug was compared and studied. The blood pressure value of one to several years after (of the same month) the use of antihypertensive drug was used for comparison.

1) Effect of antihypertensive drug on the systolic pressure (Table IA).

Table IA. The Lowering Effect on the Systolic Pressure

\begin{tabular}{|c|c|c|c|c|c|c|}
\hline \multirow{2}{*}{ Year } & \multirow{2}{*}{$\begin{array}{l}\text { Increase } \\
(+20 \mathrm{~mm} \cdot \mathrm{Hg} \\
\sim)\end{array}$} & \multirow{2}{*}{$\begin{array}{c}\text { No change } \\
( \pm 19 \mathrm{~mm} \cdot \mathrm{Hg})\end{array}$} & \multicolumn{3}{|c|}{ Decrease* } & \multirow{2}{*}{ Total } \\
\hline & & & $\begin{array}{l}(-20 \mathrm{~mm} \cdot \mathrm{Hg} \\
\sim)\end{array}$ & $\begin{array}{l}(-30 \mathrm{~mm} \cdot \mathrm{Hg} \\
\sim)\end{array}$ & $\begin{array}{l}(-40 \mathrm{~mm} \cdot \mathrm{Hg} \\
-)\end{array}$ & \\
\hline 1 & $6(7.7)$ & $49(62.8)$ & $23(29.5)$ & $9(11.5)$ & $2(2.6)$ & 78 \\
\hline 2 & $4(8.5)$ & $33(71.9)$ & $9(19.6)$ & $3(6.5)$ & $1(2.2)$ & 46 \\
\hline 3 & $2(10.0)$ & $13(65.0)$ & $5(25.0)$ & $3(15.0)$ & $2(10.0)$ & 20 \\
\hline 4 & $1(12.5)$ & $4(50.0)$ & $3(37.5)$ & $2(25.0)$ & $1(12.5)$ & 8 \\
\hline 5 & & 1 & & & & 1 \\
\hline
\end{tabular}

( ) Percentage

* The numbers in the 2 nd and 3 rd column are included in the number of the 1 st column

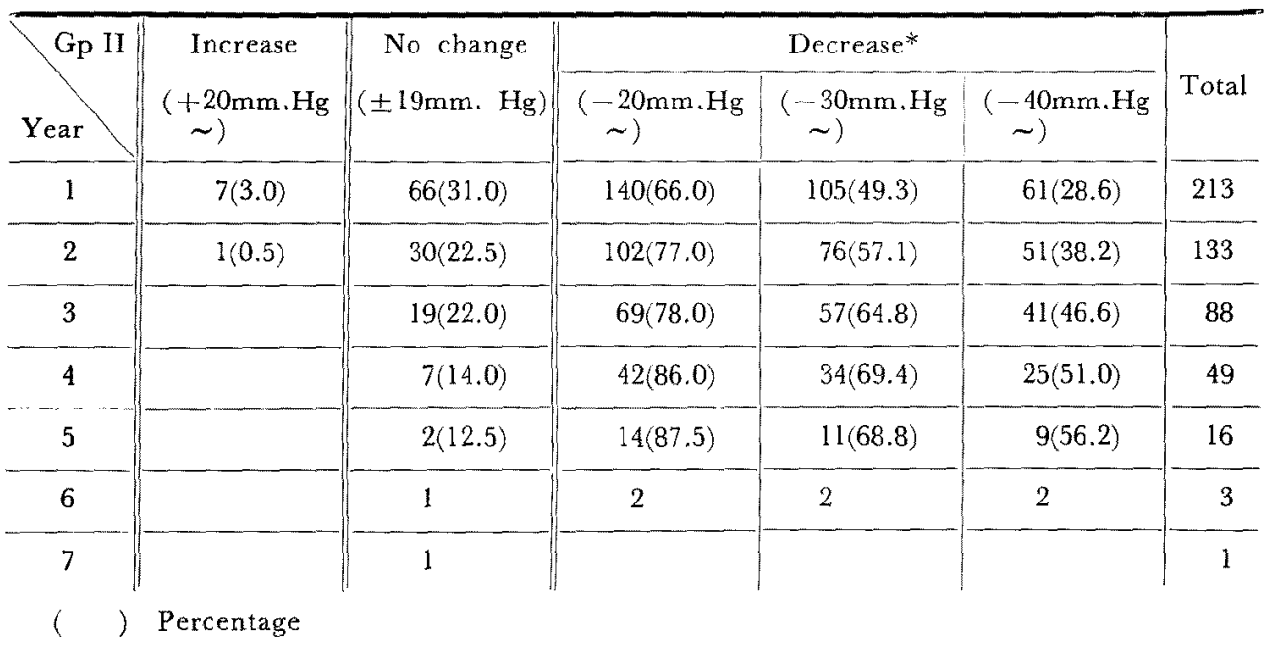




\begin{tabular}{|c|c|c|c|c|c|c|}
\hline \multirow{2}{*}{ Gp. III } & \multirow{2}{*}{$\begin{array}{c}\text { Increase } \\
(+20 \mathrm{~mm} \cdot \mathrm{Hg} \\
\sim)\end{array}$} & \multirow{2}{*}{$\mid \begin{array}{c}\text { No changc } \\
( \pm 19 \mathrm{~mm} . \mathrm{Hg})\end{array}$} & \multicolumn{3}{|c|}{ Decrease } & \multirow[b]{2}{*}{ Total } \\
\hline & & & $\left(\begin{array}{c}-20 \mathrm{~mm} \\
\sim\end{array}\right) \cdot \mathrm{Hg}$ & $\underset{\sim}{(-30 \mathrm{~mm}} \cdot \mathrm{Hg}$ & $\underset{\sim}{(-40 \mathrm{~mm} \cdot \mathrm{Hg}}$ & \\
\hline 1 & & $9(11.0)$ & $74(89.0)$ & $70(84.4)$ & $62(74.6)$ & 83 \\
\hline 2 & $2(32.0)$ & $7(11.5)$ & $52(85.3)$ & $51(83.5)$ & $49(80.4)$ & 61 \\
\hline 3 & & $2(5.6)$ & $33(94.4)$ & $32(91.5)$ & $26(74.4)$ & 35 \\
\hline 4 & & & 18 & 17 & 17 & 18 \\
\hline 5 & & & 12 & 12 & 11 & 12 \\
\hline 6 & & & 1 & 1 & 1 & 1 \\
\hline
\end{tabular}

The difference in systolic pressure of under $20 \mathrm{~mm}$. $\mathrm{Hg}$ was considered "without change" and any difference more than $20 \mathrm{~mm}$. Hg. was considered as "increased" or "decreased."

The lowering effect $(+)$ was observed significantly as the order of the group increases from Group I to Group III. This was observed in all cases, in other words, in groups with lowering of blood pressure over $20 \mathrm{~mm} . \mathrm{Hg}, 30 \mathrm{~mm} . \mathrm{Hg}$ and $40 \mathrm{~mm} . \mathrm{Hg}$. The lowering of blood pressure was increasingly evident as the period of years increase in which drug therapy was used. There are more cases "without change" in Group I, and this must be due to the fact that this group consists mainly of less severe cases and the aim in lowering the blood pressure is set only at a maximum of $20 \mathrm{~mm}$. Hg.

2) Effect of antihypertensive drug on the diastolic pressure (Table IB).

The blood pressure prior to the use of drug was compared to the blood pressure value after the use of drug, e.g., any change less than $10 \mathrm{~mm} . \mathrm{Hg}$ was considered as "No change" and any change over $10 \mathrm{~mm}$. $\mathrm{Hg}$ as "increased" or "decreased." The results obtained were similar to those of the systolic pressure.

Table IB. The Lowering Effect on the Diastolic Pressure

\begin{tabular}{|c|c|c|c|c|c|}
\hline \multirow{2}{*}{$\begin{array}{l}\text { Gp. I } \\
\text { Year }\end{array}$} & \multirow{2}{*}{$\begin{array}{c}\text { Increase } \\
(+10 \mathrm{~mm} \cdot \mathrm{Hg} \\
\sim)\end{array}$} & \multirow{2}{*}{$\begin{array}{l}\text { No change } \\
( \pm 9 \mathrm{~mm} . \mathrm{Hg})\end{array}$} & \multicolumn{2}{|c|}{ Decrease } & \multirow{2}{*}{ Total } \\
\hline & & & $(-10 \mathrm{~mm} \cdot \mathrm{Hg} \sim)$ & $(-20 \mathrm{~mm} \cdot \mathrm{Hg} \sim)$ & \\
\hline 1 & $7(7.8)$ & $54(59.3)$ & $30(32.9)$ & & 91 \\
\hline 2 & $3(5.2)$ & $30(51.7)$ & $25(43.1)$ & $7(12.1)$ & 58 \\
\hline 3 & $1(4.7)$ & $16(58.3)$ & $10(37.0)$ & $3(11.1)$ & 27 \\
\hline 4 & $1(16.7)$ & $3(50.0)$ & $2(33.3)$ & & 6 \\
\hline 5 & & $3(75.0)$ & $1(25.0\rangle$ & & 4 \\
\hline
\end{tabular}




\begin{tabular}{|c|c|c|c|c|c|}
\hline \multirow{2}{*}{ Gp.II } & \multirow{2}{*}{$\begin{array}{c}\text { Increase } \\
\left(\begin{array}{c}+10 \mathrm{~mm} \cdot \mathrm{Hg} \\
\sim)\end{array}\right.\end{array}$} & \multirow{2}{*}{$\begin{array}{l}\text { No change } \\
( \pm 9 \mathrm{~mm} . \mathrm{Hg})\end{array}$} & \multicolumn{2}{|c|}{ Decrease } & \multirow{2}{*}{ Total } \\
\hline & & & $(-10 \mathrm{~mm} \cdot \mathrm{Hg} \sim)$ & $(-20 \mathrm{~mm} . \mathrm{Hg} \sim)$ & \\
\hline 1 & $8(5.0)$ & $56(30.8)$ & $118(64.2)$ & $54(29.7)$ & 182 \\
\hline 2 & $7(6.2)$ & $31(27.4)$ & $75(66.4)$ & $41(36.5)$ & 113 \\
\hline 3 & $2(2.8)$ & $16(21.9)$ & $55(75.3)$ & $30(41.1)$ & 73 \\
\hline 4 & $2(4.6)$ & $8(19.2)$ & $32(76.2)$ & $16(38.1)$ & 42 \\
\hline 5 & & $\mathrm{I}(11.2)$ & $8(88.8)$ & $5(55.5)$ & 9 \\
\hline 6 & & & 1 & 1 & 1 \\
\hline
\end{tabular}

( ) Percentage

\begin{tabular}{|c|c|c|c|c|c|}
\hline \multirow{2}{*}{ Gear III } & \multirow{2}{*}{$\begin{array}{c}\text { Increase } \\
\left(\begin{array}{c}+10 \mathrm{~mm} \cdot \mathrm{Hg} \\
\sim)\end{array}\right.\end{array}$} & \multirow{2}{*}{$\begin{array}{l}\text { No change } \\
( \pm 9 \mathrm{~mm} . \mathrm{Hg})\end{array}$} & \multicolumn{2}{|c|}{ Decrease } & \multirow{2}{*}{ Total } \\
\hline & & & $(-10 \mathrm{~mm} \cdot \mathrm{Hg} \sim)$ & $(-20 \mathrm{~mm} \cdot \mathrm{Hg})$ & \\
\hline 1 & $2(2.0)$ & $5(5.0)$ & $92(93.0)$ & $71(71.7)$ & 99 \\
\hline 2 & & $8(12.1)$ & $58(87.9)$ & $49(75.6)$ & 66 \\
\hline 3 & & $1(2.2)$ & $43(97.8)$ & $30(68.2)$ & 44 \\
\hline 4 & & & 29 & $28(96.5)$ & 29 \\
\hline 5 & & & 18 & $17(94.5)$ & 18 \\
\hline 6 & & & 3 & 3 & 3 \\
\hline 7 & & & 1 & & 1 \\
\hline$(\quad)$ & Percentage & & & & \\
\hline
\end{tabular}

II. Seasonal comparison of the blood pressure in the summer and winter months during the use of antihypentensive drug.

The values of both systolic pressure and diastolic pressure were significantly higher in January than in July (Table II A, B).

Table II A.

\begin{tabular}{c|c|c|c|c}
\hline $\begin{array}{c}\text { Systolic } \\
\text { pressure }\end{array}$ & Group I & Group II & Group III & Total Cases \\
\hline January & $163 \mathrm{~mm} . \mathrm{Hg}$ & $170 \mathrm{~mm} . \mathrm{Hg}$ & $188 \mathrm{~mm} . \mathrm{Hg}$ & $172 \mathrm{~mm} . \mathrm{Hg}$ \\
\hline July & $156 \mathrm{~mm} . \mathrm{Hg}$ & $159 \mathrm{~mm} . \mathrm{Hg}$ & $176 \mathrm{~mm} . \mathrm{Hg}$ & $163 \mathrm{~mm} \cdot \mathrm{Hg}$ \\
& $<.001$ & $<.001$ & $<.001$
\end{tabular}


Table II B.

\begin{tabular}{l|c|c|c|c}
\hline $\begin{array}{c}\text { Diastolic } \\
\text { pressure }\end{array}$ & Group I & Group II & Group III & Total cases \\
\hline January & $85 \mathrm{~mm} \cdot \mathrm{Hg}$ & $95 \mathrm{~mm} \cdot \mathrm{Hg}$ & $107 \mathrm{~mm} \cdot \mathrm{Hg}$ & $96 \mathrm{~mm} . \mathrm{Hg}$ \\
\hline July & $83 \mathrm{~mm} \cdot \mathrm{Hg}$ & $91 \mathrm{~mm} \cdot \mathrm{Hg}$ & $98 \mathrm{~mm} \cdot \mathrm{Hg}$ & $92 \mathrm{~mm} \cdot \mathrm{Hg}$ \\
& $<.001$ & $<.001$ & $<.001$ &
\end{tabular}

III. Types of antihypertensive drug used.

The type and combination of the antihypertensive drugs used at the end of the first year of the treatment and those in the last year (the most recent year) were studied.

1) The number of antihypertensive drugs used (Table III A, B).

Regardless of the classification of the systolic and diastolic pressure, the cases receiving more than 2 types of antihypertensive drugs increase in number as the severity of the group advances. In recent years, Group III showed tendency of increase in cases receiving more than 2 types of antihypertensive drugs.

Table III A. Number of Hypertensive Drugs Used

\begin{tabular}{|c|c|c|c|c|c|c|}
\hline \multicolumn{2}{|c|}{ Systolic pressure } & 1 Drug & 2 Drugs & 3 Drugs & 4 Drugs & Total \\
\hline \multirow{2}{*}{ Gp. I } & $\begin{array}{r}\text { After } \\
1 \text { year }\end{array}$ & $73(74)$ & $25(26)$ & 0 & 0 & 98 \\
\hline & $\begin{array}{l}\text { Last } \\
\text { year }\end{array}$ & $51(63)$ & $28(35)$ & 2 & 0 & 81 \\
\hline \multirow{2}{*}{ Gp. II } & $\begin{array}{r}\text { After } \\
1 \text { year }\end{array}$ & $124(54)$ & $92(40)$ & $14(6)$ & 0 & 230 \\
\hline & $\begin{array}{l}\text { Last } \\
\text { year }\end{array}$ & $117(53)$ & $83(37)$ & $20(9)$ & 2 & 222 \\
\hline \multirow{2}{*}{ Gp. III } & $\begin{array}{r}\text { After } \\
1 \text { year }\end{array}$ & $33(40)$ & $40(48)$ & $10(12)$ & 1 & 84 \\
\hline & $\begin{array}{l}\text { Last } \\
\text { year }\end{array}$ & $24(28)$ & $41(48)$ & $19(22)$ & 2 & 86 \\
\hline
\end{tabular}

( ) Percentage

Table III B.

\begin{tabular}{|c|c|c|c|c|c|c|}
\hline \multicolumn{2}{|c|}{ Diastolic pressure } & 1 Drug & 2 Drugs & 3 Drugs & 4 Drugs & Total \\
\hline \multirow{2}{*}{ Gp. I } & $\begin{array}{l}\text { After } \\
1 \text { year }\end{array}$ & $70(75)$ & $20(21)$ & 2 & 1 & 93 \\
\hline & $\begin{array}{l}\text { Last } \\
\text { year }\end{array}$ & $61(66)$ & $26(28)$ & $5(5)$ & 1 & 93 \\
\hline \multirow{2}{*}{ Gp. II } & $\begin{array}{l}\text { After } \\
1 \text { year }\end{array}$ & $102(56)$ & $67(37)$ & $12(7)$ & & 181 \\
\hline & $\begin{array}{l}\text { Last } \\
\text { year }\end{array}$ & $102(56)$ & $65(36)$ & $15(8)$ & & 182 \\
\hline \multirow{2}{*}{ Gp. III } & $\begin{array}{l}\text { After } \\
1 \text { year }\end{array}$ & $47(47)$ & $42(42)$ & $11(11)$ & & 100 \\
\hline & $\begin{array}{l}\text { Last } \\
\text { year }\end{array}$ & $34(34)$ & $38(38)$ & $25(25)$ & 3 & 100 \\
\hline
\end{tabular}

( ) Pcrcentage 
2) The names of antihypertensive drugs used in each group (Table IV A, B).

The use of Serpentina and Hydralazine together were the most commonly used combination.

IV. The amount of antihypertensive drugs used in one year (Table V).

The average amount of antihypertensive drug used per person in one year was calculated by selecting a total of 40 cases through random sampling. Regardless of the classification, as the severity of the group advanced, the annual use of Hydralazine showed an increase. The annual use of this drug increases in the second year in comparison to the first year.

As for the use of Serpentina, expect for the smallest annual use of Serpentina in Group I according to the systolic classification, there is no specific tendency observed in different groups or in different years.

Table IV A. The Names of the Drugs Used in Each Group

\begin{tabular}{|c|c|c|c|c|c|c|c|}
\hline & Systolic pres. & \multicolumn{2}{|c|}{ Gp. I } & \multicolumn{2}{|c|}{ Gp. II } & \multicolumn{2}{|c|}{ Gp. III } \\
\hline & Antihypertensive drug & $\begin{array}{c}\text { After } \\
1 \\
\text { year }\end{array}$ & $\begin{array}{l}\text { Last } \\
\text { year }\end{array}$ & $\begin{array}{c}\text { After } \\
1 \\
\text { year }\end{array}$ & $\begin{array}{l}\text { Last } \\
\text { year }\end{array}$ & $\begin{array}{c}\text { After } \\
1 \\
\text { year }\end{array}$ & $\begin{array}{l}\text { Last } \\
\text { year }\end{array}$ \\
\hline \multirow{5}{*}{$\begin{array}{c}1 \\
\text { Drug }\end{array}$} & RS & 65 & 48 & 104 & 92 & 28 & 21 \\
\hline & AP & 1 & 1 & 11 & 11 & 4 & 1 \\
\hline & B & 0 & 2 & 3 & 2 & 1 & 2 \\
\hline & Th & 7 & 0 & 4 & 12 & 0 & 0 \\
\hline & Others & 0 & 0 & 2 & 0 & 0 & 0 \\
\hline \multirow{5}{*}{$\begin{array}{c}2 \\
\text { Drugs }\end{array}$} & $\mathrm{RS}+\mathrm{AP}$ & 19 & 19 & 52 & 54 & 25 & 28 \\
\hline & $\mathrm{RS}+\mathrm{B}$ & 1 & 0 & 19 & 14 & 11 & 6 \\
\hline & $\mathrm{RS}+\mathrm{Th}$ & 4 & 8 & 6 & 13 & 1 & 5 \\
\hline & $\mathrm{RS}+\mathrm{V}$ & 0 & 0 & 4 & 0 & 3 & 0 \\
\hline & Others & 1 & 1 & 1 & 2 & 0 & 2 \\
\hline \multirow{4}{*}{$\begin{array}{c}3 \\
\text { Drugs }\end{array}$} & $\mathrm{RS}+\mathrm{AP}+\mathrm{B}$ & 0 & 1 & 9 & 7 & 8 & 9 \\
\hline & $\mathrm{RS}+\mathrm{AP}+\mathrm{Th}$ & 0 & 1 & 4 & 10 & 1 & 4 \\
\hline & $\mathrm{RS}+\mathrm{B}+\mathrm{Th}$ & 0 & 0 & 1 & 3 & 0 & 5 \\
\hline & Others & 0 & 0 & 0 & 0 & 1 & 1 \\
\hline $\begin{array}{c}4 \\
\text { Drugs }\end{array}$ & $\mathrm{RS}+\mathrm{AP}+\mathrm{B}+\mathrm{Th}$ & 0 & 0 & 0 & 2 & 1 & 2 \\
\hline
\end{tabular}


Table IV B.

\begin{tabular}{|c|c|c|c|c|c|c|c|}
\hline & Diastolic pres. & \multicolumn{2}{|c|}{ Gp. I } & \multicolumn{2}{|c|}{ Gp. II } & \multicolumn{2}{|c|}{ Gp. III } \\
\hline & Antihypertensive drug & $\begin{array}{c}\text { After } \\
\text { year }\end{array}$ & $\begin{array}{l}\text { Last } \\
\text { year }\end{array}$ & $\begin{array}{c}\text { After } \\
\perp \\
\text { year }\end{array}$ & $\begin{array}{l}\text { Last } \\
\text { year }\end{array}$ & $\begin{array}{c}\text { After } \\
1 \\
\text { year }\end{array}$ & $\begin{array}{l}\text { Last } \\
\text { year }\end{array}$ \\
\hline \multirow{5}{*}{$\begin{array}{c}1 \\
\text { Drug }\end{array}$} & $\mathrm{RS}$ & 65 & 46 & 90 & 79 & 41 & 26 \\
\hline & AP & 4 & 3 & 8 & 9 & 3 & 4 \\
\hline & B & & 1 & & 3 & 3 & 1 \\
\hline & Th & 1 & 7 & 3 & 9 & & 3 \\
\hline & Others & & 4 & 1 & 1 & & \\
\hline \multirow{5}{*}{$\begin{array}{c}2 \\
\text { Drugs }\end{array}$} & $\mathrm{RS}+\mathrm{AP}$ & 16 & 21 & 43 & 46 & 25 & 23 \\
\hline & $\mathrm{RS}+\mathrm{B}$ & 1 & 1 & 9 & 7 & 12 & 5 \\
\hline & $\mathrm{RS}+\mathrm{Th}$ & 1 & 4 & 9 & 12 & 3 & 8 \\
\hline & $\mathrm{RS}+\mathrm{V}$ & 2 & & 3 & & 1 & \\
\hline & Others & & & 3 & & 1 & 2 \\
\hline \multirow{4}{*}{$\begin{array}{c}3 \\
\text { Drugs }\end{array}$} & $\mathrm{RS}+\mathrm{AP}+\mathrm{B}$ & 2 & 1 & 8 & 7 & 6 & 7 \\
\hline & $\mathrm{RS}+\mathrm{AP}+\mathrm{Th}$ & & 4 & 2 & 6 & 2 & 10 \\
\hline & $\mathrm{RS}+\mathrm{B}+\mathrm{Th}$ & & & 1 & 1 & 2 & 7 \\
\hline & Others & & & 1 & 1 & 1 & 1 \\
\hline $\begin{array}{c}4 \\
\text { Drugs }\end{array}$ & $\mathrm{RS}+\mathrm{AP}+\mathrm{B}+\mathrm{Th}$ & 1 & 1 & & & & 3 \\
\hline
\end{tabular}

Remarks: RS: Serpentina

AP: Apresoline

B : Ganglionic blocking agent

Th: Chlorothiazide

$\mathrm{V}$ : Veriloid

V. The seasonal comparison of the average amount of antihypertensive drugs used per day person (Table VI).

The comparison was made of the antihypertensive drug used in the summer and winter season, represented by the amount used in the month of July and January. With 2 or 3 exceptions, the average amount used is somewhat higher in the winter in comparison to the summer months. 
Table V. The Average Amount of Antihypertensive Drug Used in One Yyar

(Observation of 40 random sample cases)

\begin{tabular}{|c|c|c|c|c|c|c|c|c|c|c|c|}
\hline \multicolumn{2}{|l|}{ Systolic } & $\mathrm{R}$ & RS & AP & $\begin{array}{l}\text { Veri- } \\
\text { loid }\end{array}$ & $\mathrm{C}_{6}$ & $\mathrm{C}_{5}$ & $\begin{array}{l}\text { Pem- } \\
\text { pidine }\end{array}$ & $\mathrm{Th}$ & $\mathrm{H}-\mathrm{Th}$ & HFT \\
\hline \multirow{4}{*}{$\begin{array}{l}\text { First } \\
\text { year }\end{array}$} & Gp. I & 66 & 610 & 658 & & & & & & 667 & 667 \\
\hline & Gp. II & 136 & 908 & 11,127 & & 7.5 & 13.6 & & 2.4 & & 273 \\
\hline & Gp. III & 172 & 452 & 15,978 & 115 & 55.0 & & & & & \\
\hline & Total & 128 & 738 & 9,863 & 63 & 16.5 & 7.5 & & 18 & 17 & 150 \\
\hline $\begin{array}{l}\text { Second } \\
\text { year }\end{array}$ & Total & 114 & 580 & 17,800 & 76 & 16.0 & & 2.9 & 1.7 & & \\
\hline
\end{tabular}

\begin{tabular}{|c|c|c|c|c|c|c|c|c|c|c|c|}
\hline \multicolumn{2}{|c|}{ Diastolic pressure } & $\mathrm{R}$ & $\begin{array}{l}\text { RS } \\
\text { mg. }\end{array}$ & $\begin{array}{l}\text { AP } \\
\mathrm{mg} .\end{array}$ & $\begin{array}{l}\text { Veri- } \\
\text { loid }\end{array}$ & $\begin{array}{c}\mathrm{C}_{6} \\
\mathrm{Gm} .\end{array}$ & $\mathrm{C}_{5}$ & $\begin{array}{l}\text { Pem- } \\
\text { pidine }\end{array}$ & $\begin{array}{r}\mathrm{Th} \\
\mathrm{Gm} .\end{array}$ & $\begin{array}{l}\mathrm{H}-\mathrm{Th} \\
\mathrm{mg} .\end{array}$ & $\begin{array}{c}\mathrm{HFT} \\
\text { mg. }\end{array}$ \\
\hline \multirow{4}{*}{$\begin{array}{l}\text { First } \\
\text { year }\end{array}$} & Gp. I & 111 & 744 & 2,370 & & & & & & & \\
\hline & Gp. II & 123 & 470 & 8,827 & & 59.8 & 3,970 & & 7 & & \\
\hline & Gp. III & 87.6 & 1,062 & 13,440 & & 2.4 & 7,940 & & 12.5 & & 1,500 \\
\hline & Total & 115 & 686 & 7,833 & & 30.5 & 1,985 & & 6.3 & & \\
\hline $\begin{array}{l}\text { Second } \\
\text { year }\end{array}$ & Total & 142 & 584 & 11,630 & & 30.6 & 3,548 & & 4.0 & & \\
\hline
\end{tabular}

R: Reserpine RS: Raw Serpentina AP: Apresoline Th: Chlorothiazide H-Th: Hydrochlorothiazide HFT: Hydroflumethiazide

VI. The subjective symptoms of hypertension and the effect of the antihypertensive drug upon these symptoms (Table VII).

Among the subjective symptoms observed, the most commonly observed symptoms (over 20\%) according to frequency were as follows: headache, stiffness of the shoulder, heaviness of the head, dizziness and palpitation. Symptoms which showed high percentage of improvement (over $60 \%$ ) were flushed feeling, insomnia, numbness, shortness of breath and anginal pain. 
Table VI.

\begin{tabular}{|c|c|c|c|c|c|c|c|}
\hline \multirow{2}{*}{\multicolumn{2}{|c|}{ Name of drug }} & \multicolumn{2}{|c|}{ Group I } & \multicolumn{2}{|c|}{ Group II } & \multicolumn{2}{|c|}{ Group III } \\
\hline & & January & July & January & July & January & July \\
\hline Reserpine & $\mathrm{mg}$. & 0.37 & 0.30 & 0.36 & 0.35 & 0.53 & 0.48 \\
\hline Raw Serpentita & $\mathrm{mg}$. & 2.1 & 2.0 & 1.9 & 1.7 & 1.6 & 1.5 \\
\hline Apresoline & $\mathrm{mg}$. & 24.0 & 16.2 & 28.9 & 29.1 & 68.4 & 55.1 \\
\hline Veriloid & $\mathrm{mg}$. & & & 0.1 & 0.13 & 0.13 & 0.18 \\
\hline $\mathrm{C}_{6}$ & $\mathrm{Gm}$ & 0.01 & & 0.67 & 0.1 & 0.22 & 0.2 \\
\hline $\mathrm{C}_{5}$ & $\mathrm{mg}$. & 0.36 & & 6.2 & 4.3 & 20.4 & 13.5 \\
\hline Mecamylamine & $\mathrm{mg}$. & & & & & 0.39 & 0.39 \\
\hline Pempidine & mg. & & & 0.05 & 0.06 & 0.04 & 0.05 \\
\hline Th & Gm. & 0.023 & 0.014 & 0.002 & 0.015 & 1.39 & 0.02 \\
\hline H-Th & mg. & & 1.4 & 0.4 & 0.8 & 1.0 & 0.3 \\
\hline HFT & ng. & 1.5 & 3.3 & 0.8 & 3.0 & & 1.2 \\
\hline
\end{tabular}

\begin{tabular}{|c|c|c|c|c|c|c|}
\hline \multirow{2}{*}{ Diastolic pres. } & \multicolumn{2}{|c|}{ Group I } & \multicolumn{2}{|c|}{ Group II } & \multicolumn{2}{|c|}{ Group III } \\
\hline & January & July & January & July & January & July \\
\hline Reserpine mg. & 0.35 & 0.33 & 0.39 & 0.38 & 0.52 & 0.47 \\
\hline Raw Serpentina ${ }^{\mathrm{mg} .}$ & 2.0 & 2.0 & 2.0 & 1.8 & 1.7 & 1.6 \\
\hline Apresoline $\quad \mathrm{mg}$. & 20.0 & 26.9 & 34.0 & 30.4 & 55.0 & 51.0 \\
\hline Veriloid & 0.10 & 0.13 & 0.11 & 0.14 & 0.07 & 0.13 \\
\hline $\mathrm{Gm}$ & 0.03 & 0.02 & 0.11 & 0.07 & 0.22 & 0.24 \\
\hline $\mathrm{mg}$ & 0.025 & 0.022 & 3.0 & 2.90 & 22.5 & 12.7 \\
\hline Mecamylamine mg. & & & & & 0.33 & 0.28 \\
\hline Pempidine $\quad \mathrm{mg}$. & & & 0.01 & 0.01 & 0.05 & 0.04 \\
\hline Gm. & 0.02 & 0.01 & 0.03 & 0.01 & 0.02 & 0.02 \\
\hline $\mathrm{H}-\mathrm{Th}$ & & 0.30 & 0.5 & 0.8 & 0.7 & 1.0 \\
\hline HFT & & 1.5 & 1.6 & 3.2 & & 3.3 \\
\hline
\end{tabular}


VII. The effect of antihypertensive drug upon the findings of the various organs (Table VIII A, B).

No conclusion can be drawn concerning this subject since the data from the tests were incomplete due to the fact that the cases studied were all out-patients. However, it was implicated that there were cases which showed improvement in the findings of the urine, EKG, X-ray of the heart and the eyeground. On the other hand, there were cases in which the condition became worse despite the treatment.

Table VII.

On 379 cases

\begin{tabular}{|c|c|c|c|c|c|}
\hline & $\begin{array}{l}\text { Chief } \\
\text { complaint }\end{array}$ & Improvement & No change & $\begin{array}{c}\text { Became } \\
\text { worse }\end{array}$ & Unknown \\
\hline Headache & $142(37.5)$ & $96(67.5)$ & $31(21.8)$ & 1 & $14(9.9)$ \\
\hline $\begin{array}{l}\text { Heaviness of } \\
\text { the head }\end{array}$ & $102(26.9)$ & $70(68.6)$ & $26(25.5)$ & 0 & 6 \\
\hline Dizziness & $99(26.1)$ & $68(68.7)$ & $24(24.2)$ & 1 & 6 \\
\hline $\begin{array}{l}\text { Ringing of the } \\
\text { ear }\end{array}$ & $56(14.8)$ & $38(67.8)$ & $16(28.6)$ & 0 & 2 \\
\hline Flushed feeling & $37(9.8)$ & $27(73.0)$ & 6 & 0 & 4 \\
\hline Forgetfulness & 9 & 4 & 4 & 0 & 1 \\
\hline Irritability & $12(3.2)$ & 8 & 3 & 1 & 0 \\
\hline Insomnia & $52(13.7)$ & $35(67.4)$ & $11(21.2)$ & 2 & 4 \\
\hline $\begin{array}{l}\text { Stiffness of the } \\
\text { shoulder }\end{array}$ & $113(29.8)$ & $67(59.3)$ & $36(31.8)$ & 3 & 7 \\
\hline Numbness & $40(10.5)$ & $24(60.0)$ & $11(27.5)$ & 0 & 5 \\
\hline Epistaxis & 9 & 5 & 4 & 0 & 0 \\
\hline $\begin{array}{l}\text { Visual } \\
\text { disturbance }\end{array}$ & $11(2.9)$ & 7 & 3 & 1 & 0 \\
\hline Palpitation & $86(22.7)$ & $63(73.3)$ & $\mathrm{I} 3(15.2)$ & 3 & 8 \\
\hline $\begin{array}{l}\text { Shortness of } \\
\text { breath }\end{array}$ & $67(17.7)$ & $45(67.2)$ & $13(19.4)$ & 4 & 5 \\
\hline Anginal pain & $33(8.7)$ & $22(65.5)$ & 8 & 2 & 1 \\
\hline Edema & $43(11.3)$ & $21(48.8)$ & $19(44.2)$ & 2 & 1 \\
\hline Nocturia & $14(3.7)$ & 9 & 3 & 0 & 2 \\
\hline
\end{tabular}


VIII. The percentage of occurrence of complications during the treatment with antihypertensive drug (Table IX A, B).

The occurrence of cerebral, cardiac and renal complications were studied by comparing the period before the use of antihypertensive drug and during the period in which antihypertensive drugs were used. In general, it was observed that the occurrence of cerebral and cardiac

Table VIII A.

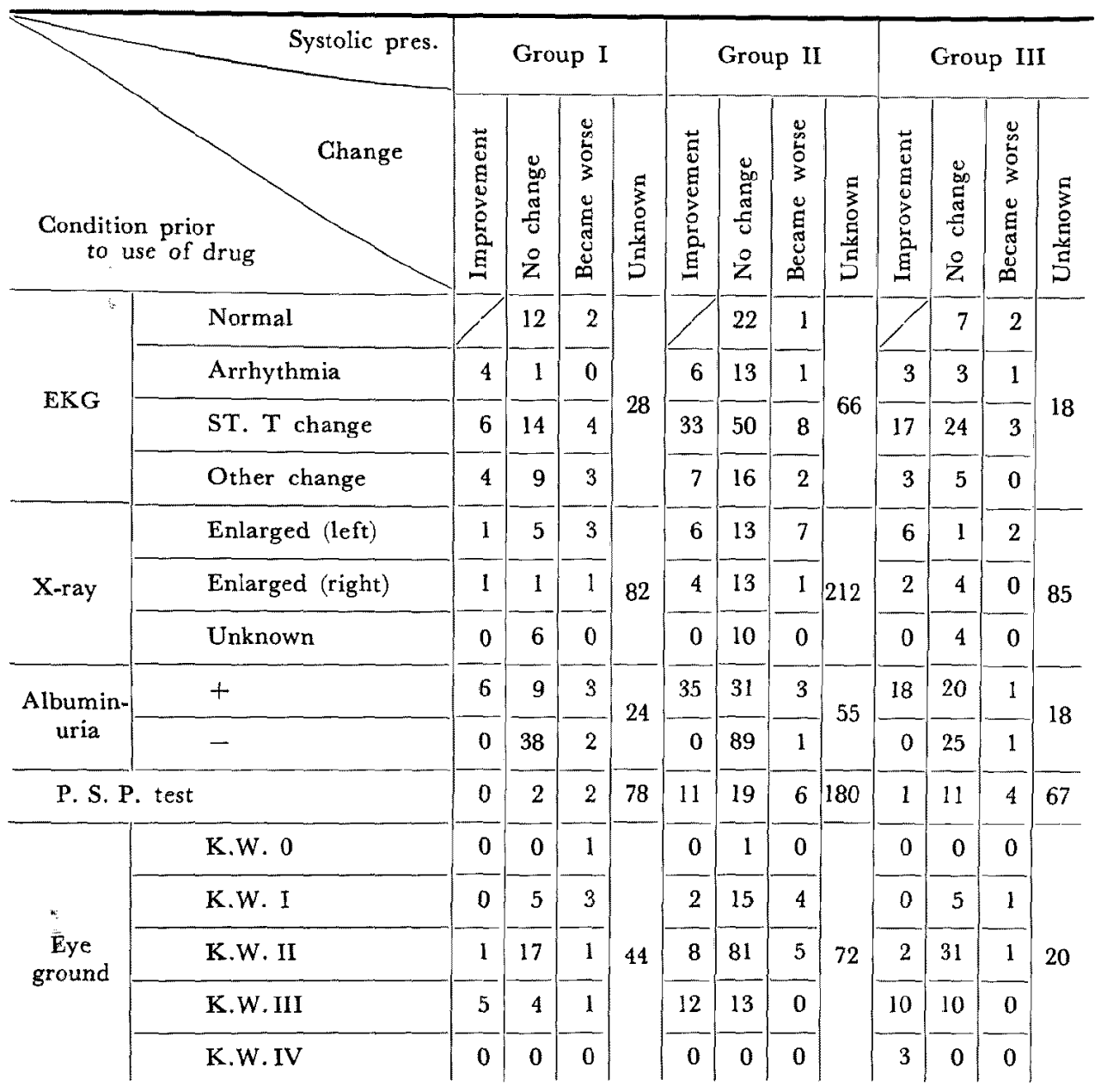


complications was less during the period in which the drugs were used. However, no conclusion can be drawn, because of the incomplete information of cases with complication who have discontinued to come to the clinic. In Group III of both systolic and diastolic classification there was no difference observed in the occurrence of cerebral vascular accident prior to or during the use of antihypertensive drug.

Table VIII B.

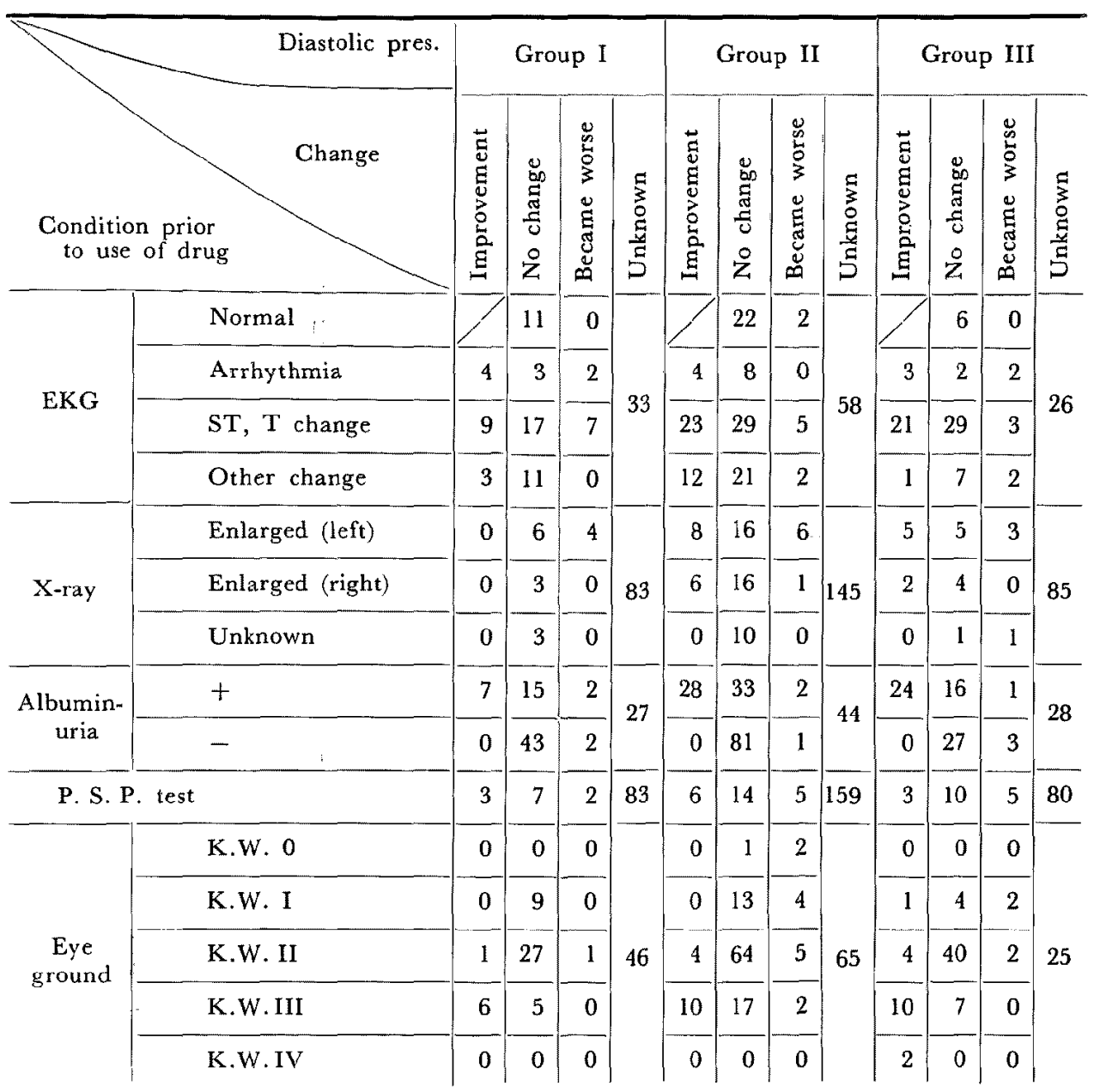


Table IX A. Occurrence of Complication in Cases Receiving antihypertensive Drug for a Long Period

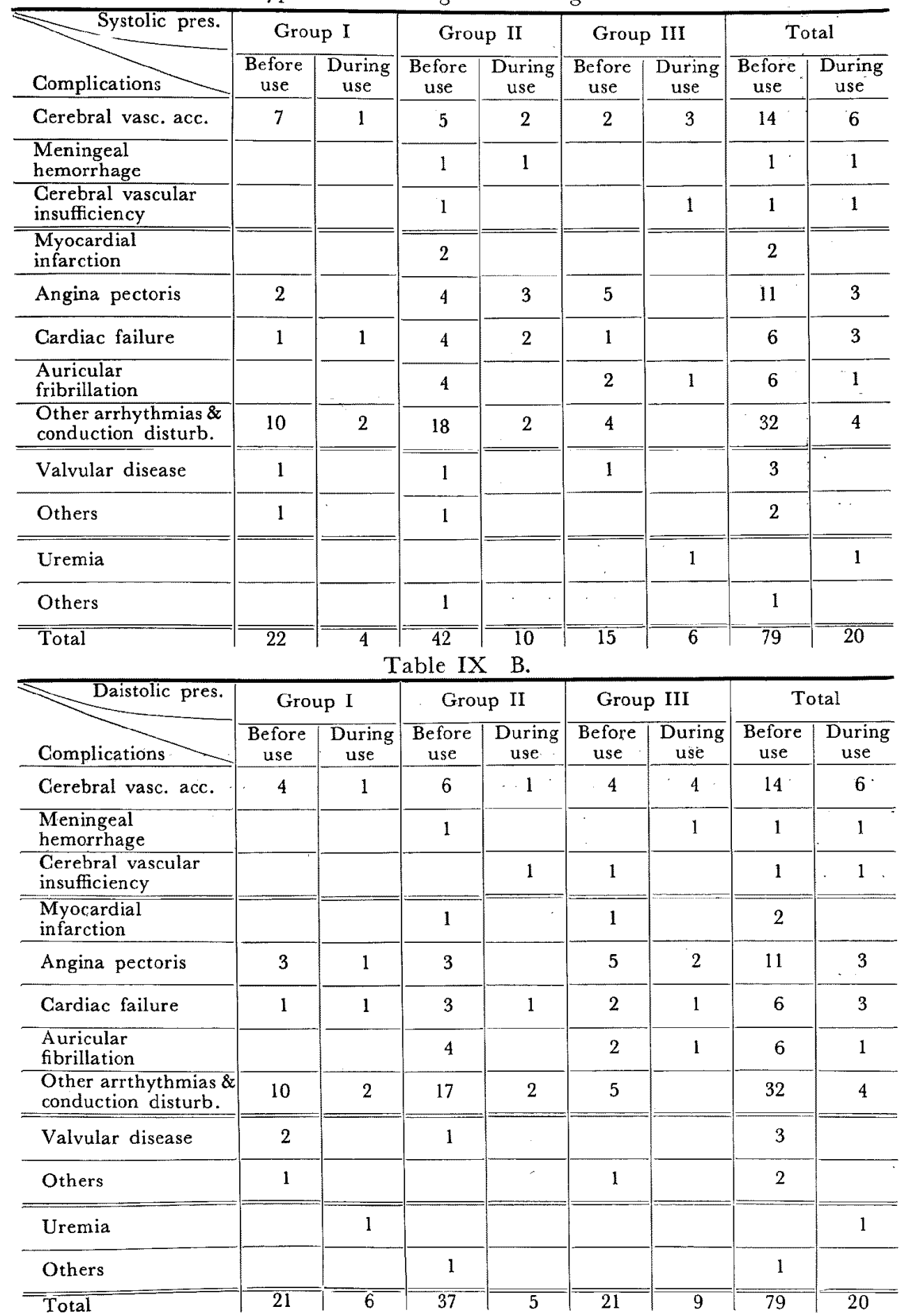


Table X. Side Effects of Antihypertensive Drug

\begin{tabular}{|c|c|c|c|c|c|}
\hline Antihypertensive Drug & RS & AP & B & $\mathrm{Th}$ & Total \\
\hline Stuffed nose & 60 & 2 & & & 62 \\
\hline Myalgia \& neuralgia & 13 & 3 & 2 & 1 & 19 \\
\hline Anorexia & 9 & 5 & 1 & 4 & 19 \\
\hline Diarrhea & 7 & 3 & & 2 & 12 \\
\hline Constipation & 14 & 3 & 10 & & 27 \\
\hline Cerebral svmptoms & 4 & 2 & 3 & & 9 \\
\hline Exanthema & 2 & & 1 & & 3 \\
\hline Hrpokalemia & & & & 1 & 1 \\
\hline Others & 6 & 5 & 3 & 2 & 16 \\
\hline Total & 115 & 23 & 20 & 10 & 168 \\
\hline
\end{tabular}

Remarks: RS: Serpentina

AP: Apresoline

B : Ganglionic blocking agent

Th: Chlorothiazide

\section{SUMmary}

(1) According to the survey of 379 patients who received treatment with antihypertensive drugs for more than one year, considerable effect of lowering blood pressure was observed in general with the improvement of subjective symptoms. There were also improvements in the objective signs in some cases.

(2) In advanced hypertensive cases (systolic pressure over $220 \mathrm{~mm} . \mathrm{Hg}$ and diastolic pressure over $120 \mathrm{~mm} . \mathrm{Hg}$ ) the occurrence of crebral vascular accidents were higher than in hypertensive cases with lower blood pressure in spite of the fact that they were under treatment with hypertensive drugs.

(3) In order to know the present situation of the treatment with antihypertensive drugs, the type of drug used, the average amount used per person per year and the average amount of usage per person per day in the summer and winter season were studied. 